\title{
Corifollitropin alfa compared to daily rFSH or HP-HMG in GnRH antagonist controlled ovarian stimulation protocol for patients undergoing assisted reproduction
}

\author{
Priscila Morais Galvão Souza1, Bruno Ramalho de Carvalho ${ }^{1}$, Hitomi Miura Nakagawa ${ }^{1}$, Thalita Reis Esselin \\ Rassi $^{1}$, Antônio César Paes Barbosa ${ }^{1}$, Adelino Amaral Silva ${ }^{1}$ \\ ${ }^{1}$ GENESIS - Center for Assistance in Human Reproduction, Brasília, DF, Brazil.
}

\begin{abstract}
Objective: This study aimed to compare the outcomes of controlled ovarian stimulation (COS) with corifollitropin alfa versus daily recombinant follicle-stimulating hormone (rRFSH) or highly purified human menopausal gonadotropin (HP-HMG) in patients undergoing in vitro fertilization (IVF) cycles based on gonadotropin-releasing hormone ( $\mathrm{GnRH}$ ) antagonist protocols. The primary endpoints were total number of oocytes and mature oocytes.

Methods: This retrospective study looked into 132 controlled ovarian stimulation cycles from IVF or oocyte cryopreservation performed in a private human reproduction center between January 1 and December 31,2014 . Enrollment criteria: women aged < 40 years submitted to COS with corifollitropin alfa $100 \mu \mathrm{g}$ or $150 \mu \mathrm{g}$ $(n=26)$ and rFSH or HP-HMG in the first seven days of treatment with daily doses of 150-225 IU $(n=106)$; all subjects were on GnRH antagonist protocols.

Results: The groups had similar mean ages and duration of stimulation. The mean number \pm standard deviation of total aspirated oocytes and MII oocytes was $11.9 \pm 10$ and $10.3 \pm 7.9$ in the corifollitropin alfa group, and $10.9 \pm 7.2$ and $8.6 \pm 5.7$ in the group on rFSH or HMG $(p>0.05)$. There were no significant differences in fertilization $(76.9 \%$ vs. $76.8 \%, p=1.0)$, biochemical pregnancy ( $66.7 \%$ vs. $47.2 \%$, $p=0.1561)$ or embryo implantation rates $(68.7 \%$ vs. $50 \%$, $p=0.2588$ ) between the groups using corifollitropin alfa and $\mathrm{rFSH}$ or HMG, respectively.

Conclusions: Corifollitropin alfa seems to be as effective as rFSH or HP-HMG when used in the first seven days of ovulation induction for patients undergoing assisted reproduction in $\mathrm{GnRH}$ antagonist protocols.
\end{abstract}

Keywords: Corifollitropin alfa, gonadotropins, ovulation induction, in vitro fertilization, assisted reproductive technology

\section{INTRODUCTION}

Assisted reproductive treatments often take a significant financial and emotional toll on patients, not to mention the time-consuming visits required during ovarian stimulation and the frustration inherent to a diagnosis of infertility. Standard protocols for controlled ovarian stimulation usually include daily self-administered injectable doses of gonadotropin, which increase the need for medical attention and introduce additional psychological distress as described by infertile couples; these factors combined increase the number of patients dropping out of therapy (Rajkhowa, 2006).

In such context, innovative strategies are needed to diminish the emotional stress caused by in vitro fertilization (IVF), with the development of patient-friendly, costeffective, good quality ovarian stimulation protocols (de
Carvalho, 2016). Decreasing the number of daily injections might mitigate the negative impact on the treated couple, improving cooperation and compliance, and maximizing results by reducing potential administration errors (Devroey et al., 2009).

In the presence of high affinity to FSH receptors and sustained follicle-stimulating activity, it has been proven that corifollitropin alfa is able to replace the first seven daily doses of any rFSH preparation in controlled ovarian hyperstimulation prior to IVF (Bouloux et al., 2001). Although experience with corifollitropin alfa is still incipient when compared to other gonadotropins, several studies have been carried out in recent years to assess its efficacy and compare it against traditional ovarian induction regimens (Devroey et al., 2009; Mahmoud Youssef et al., 2012; Kolibianakis et al., 2015; Griesinger et al., 2016).

This study aimed to compare the response to controlled ovarian stimulation (COS) with corifollitropin alfa, and recombinant follicle-stimulating hormone ( $\mathrm{rFSH}$ ) or highly purified human menopausal gonadotropin (HP-HMG) during the first seven days in patients on GnRH antagonist protocols offered in vitro fertilization with intracytoplasmic sperm injection (IVF/ICSI) or oocyte cryopreservation.

\section{MATERIAL AND METHODS}

This retrospective study included 307 COS cycles carried out between January 1 and December 31, 2014. All patients were recruited from the GENESIS Center for Assistance in Human Reproduction in Brasília, Brazil. Participants had to meet the following enrollment criteria: $\mathrm{COS}$ performed with either corifollitropin alfa and rFSH or HP-HMG in GnRH antagonist protocols for purposes of oocyte cryopreservation or IVF/ICSI. In the IVF/ICSI cycles, the oocytes were fertilized with sperm from the patient's partner collected from fresh semen samples; only fresh embryo transfers were considered. Cycles with oocytes submitted to preimplantation genetic diagnosis $(n=5)$, patients with age $\geq 40$ years $(n=144)$, and cycles with donated oocytes $(n=26)$ were excluded.

The treatment protocols described in the ENGAGE (Devroey et al., 2009) and ENSURE (Corifollitropin alfa Ensure Study Group, 2010) trials were adopted in this study. The patients were given either a single dose of $100 \mu \mathrm{g}(<60 \mathrm{~kg})$ or $150 \mu \mathrm{g}(\geq 60 \mathrm{~kg})$ of corifollitropin alfa (Elonva, Schering-Plough, Brazil) or daily 200-300 IU rFSH (follitropin beta, Puregon, Schering-Plough, Brazil) on day 2 or 3 of the menstrual cycle; follitropin alfa, Gonal-f, Merck, Brazil) or daily urinary HP-HMG (menotropin, Menopur, Ferring, Brazil) was administered for the first seven days of COS, followed by daily 200-300 IU rFSH or HP-HMG in a GnRH antagonist (ganirelix, Orgalutran, Schering-Plough, Brazil or cetrorelix, Cetrotide, Merck, Brazil) regimen until final follicular maturation with human chorionic gonadotropin (hCG). The primary endpoints were the total number of oocytes and mature oocytes yielded. 
The secondary endpoints were fertilization, biochemical pregnancy, and implantation rates.

The Institution's Clinical Committee approved the study. Enrolled patients gave written consent to undergo assisted reproduction technology treatment and oral consent to having their data used in the study. A specific written informed consent form was not required in this study, since research data were collected exclusively from patient files.

Statistical analysis was performed on software package GraphPad Prism version 5.00 (GraphPad Software, Inc, 2007). Samples with a normal distribution were treated with the unpaired $t$-test; the Mann-Whitney test was used for samples with non-parametric distributions. Fisher's exact test was used in contingency analysis. The level of significance was set at $p<0.05$.

\section{RESULTS}

A total of 132 patients were treated in our study; 26 subjects were given a single dose of corifollitropin alfa and 106 subjects were administered daily rFSH or HPHMG for the first seven days of COS. Table 1 describes the characteristics of the patients from each of the groups.

The mean number of oocytes and MII oocytes was not different between the groups given corifollitropin alfa and $\mathrm{rFSH}$ or HP-HMG. No differences were found in terms of fertilization rates, number of transferred embryos, biochemical pregnancy rates or embryo implantation rates between patients on corifollitropin alfa and rFSH or HPHMG (Table 2).

Table 1. Patient characteristics per treatment group

\begin{tabular}{|l|c|c|c|}
\hline & $\begin{array}{c}\text { Corifollitropin } \\
\text { alfa }\end{array}$ & $\begin{array}{c}\text { rFSH or HP- } \\
\text { HMG }\end{array}$ & $\boldsymbol{P}$ \\
\hline $\mathrm{n}$ & 26 & 106 & \\
\hline Age (years) & $34.23 \pm 4.053$ & $34.17 \pm 3.801$ & $\mathrm{NS}$ \\
\hline $\begin{array}{l}\text { Duration of } \\
\text { stimulation } \\
\text { (days) }\end{array}$ & $11.92 \pm 1.896$ & $11.87 \pm 2.168$ & NS \\
\hline
\end{tabular}

Age and duration of stimulation are expressed as means \pm standard deviations; NS = not significant

\section{DISCUSSION}

New technologies have been introduced in the realm of assisted reproduction within the last two decades. Outcomes have been improved for specific groups of patients, but none of such innovations seemed to benefit the infertile population in general. Innovations in assisted reproduction have moved toward patient-friendliness and cost-effectiveness (de Carvalho, 2016). If corifollitropin alfa and daily gonadotropins are proven equivalent in terms of effectiveness and safety, enhancements in patient-friendliness may decrease the number of patients abandoning treatment and even turn the therapy into an attractive first choice of ovulation induction.

The ENGAGE Study was a double-blind randomized clinical trial that enrolled 1,509 women in the United States and 20 European countries to compare the use of $150 \mu \mathrm{g}$ of corifollitropin alfa during the first week of stimulation versus rFSH in daily doses of $200 \mathrm{IU}$, both in antagonist protocols. The study conducted by Devroey et al. (2009) showed that corifollitropin alfa and daily rFSH had a similar pregnancy rate outcome in normal responders.

In the following year, the ENSURE Study - also a randomized double-blind trial - enrolled 396 women
Table 2. Clinical outcomes of cycles using corifollitropin alfa, and recombinant follicle-stimulating hormone (rRFSH) or highly purified human menopausal gonadotropin (HP-HMG)

\begin{tabular}{|c|c|c|c|}
\hline & $\begin{array}{c}\text { Corifollitropin } \\
\text { alfa }\end{array}$ & $\begin{array}{c}\text { rFSH/HP- } \\
\text { HMG }\end{array}$ & $\boldsymbol{P a}$ \\
\hline $\begin{array}{l}\text { Oocytes yielded, } \\
\text { total } \\
(\text { mean } \pm S D)\end{array}$ & $11.99 \pm 10$ & $10.9 \pm 7.2$ & NS \\
\hline $\begin{array}{l}\text { Oocytes yielded, } \\
\text { MII } \\
(\text { mean } \pm \text { SD })\end{array}$ & $10.3 \pm 7.9$ & $8.6 \pm 5.7$ & NS \\
\hline Fertilization, \% & 76.9 & 76.8 & NS \\
\hline $\begin{array}{l}\text { Embryos } \\
\text { transferred } \\
(\text { mean } \pm S D)\end{array}$ & $1.63 \pm 0,84$ & $1.76 \pm 0.94$ & NS \\
\hline $\begin{array}{l}\text { Biochemical } \\
\text { pregnancy, \% }\end{array}$ & 66.7 & 47.2 & NS \\
\hline $\begin{array}{l}\text { Implantation } \\
\text { rate, \% }\end{array}$ & 68.7 & 50 & NS \\
\hline
\end{tabular}

NS $=$ not significant

a Statistical analysis performed by unpaired $t$-test (normal distribution) or Mann-Whitney test (non-parametric distribution).

weighing up to $60 \mathrm{~kg}$ submitted to ovarian stimulation for IVF using a single-dose of corifollitropin alfa $100 \mu \mathrm{g}$ or daily rFSH $150 \mathrm{IU}$ for the first seven days on antagonist protocols. The study showed that corifollitropin alfa was potentially a simpler protocol for normal responders (Corifollitropin alfa Ensure Study Group, 2010).

Another randomized clinical trial comparing corifollitropin alfa and daily rFSH revealed that the number of oocytes yielded and pregnancy rates were similar for early or normal responders, regardless of treatment group (Mardešič et al., 2014). However, a recent study suggested that corifollitropin alfa may lead to a greater number of retrieved oocytes and more cancelled cycles due to ovarian hyperstimulation when compared to rFSH (Mahmoud Youssef et al., 2012).

Given the existence of adequate levels of follicular response, patient-friendliness is a relevant factor in the choice of a stimulation protocol. Women previously treated with rFSH who received corifollitropin alfa in a new cycle reported greater satisfaction with the single dose protocol, confirming that ovulation induction regimen might reduce the stress of treatment (Requena et al., 2013). In this same study, there were no significant differences between groups in areas such as implantation rate $(39.1 \%$ for corifollitropin vs. $38.4 \%$ for daily $\mathrm{rFSH}$ ) or pregnancy rate ( $45.9 \%$ for corifollitropin vs. $44.4 \%$ for daily rFSH).

Our results must be considered with caution, since the biases inherent to open non-randomized retrospective studies cannot be ruled out. Although no significant differences have been reported in the literature in reproductive outcomes between follitropin alfa and beta (Kolibianakis et al., 2015), or menotropin (Westergaard et al., 2011), there may be differences between results in fixed and flexible GnRH antagonist regimens (Kolibianakis et al., 2003), which were not analyzed as separate groups in our study. Moreover, variable daily gonadotropin doses used for stimulation may lead to different outcomes, especially on the number of gametes retrieved. Finally, according to a considerable number of references, GnRH agonist protocols are the first choice for women with good prognoses instead of $\mathrm{GnRH}$ antagonists (Orvieto et al., 
2008; Orvieto \& Patrizio, 2013), but data on corifollitropin alfa in GnRH agonist protocols are scarce, and impede further comparisons.

Corifollitropin alfa seems to be as effective as rFSH or HP-HMG in the first seven days of treatment for normal responders undergoing assisted reproduction cycles in a $\mathrm{GnRH}$ antagonist regimen.

\section{ACKNOWLEDGEMENTS}

The authors would like to thank Íris de Oliveira Cabral, $\mathrm{BSc}$, for the invaluable help with data collection and treatment, and David Barreira Gomes Sobrinho, MD, MSc, for having proofread this paper and given technical suggestions on its content.

\section{CONFLICT OF INTERESTS}

The authors have no conflicts of interest to report.

\section{Corresponding author:}

Bruno Ramalho de Carvalho

E-mail: brunoramalho@hotmail.com

Hitomi Miura Nakagawa

GENESIS - Center for Assistance in Human Reproduction Brasília, DF, Brazil

E-mail: naka.miuramd@gmail.com

\section{REFERENCES}

Bouloux PM, Handelsman DJ, Jockenhövel F, Nieschlag E, Rabinovici J, Frasa WL, de Bie JJ, Voortman G, Itskovitz-Eldor J; FSH-CTP study group. First human exposure to FSH-CTP in hypogonadotrophic hypogonadal males. Hum Reprod. 2001;16:1592-7. PMID: 11473948 DOI: https://doi.org/10.1093/humrep/16.8.1592

Corifollitropin alfa Ensure Study Group. Corifollitropin alfa for ovarian stimulation in IVF: a randomized trial in lowerbody-weight women. Reprod Biomed Online. 2010;21:6676. PMID: 20483664 DOI: 10.1016/j.rbmo.2010.03.019

de Carvalho BR. Often times, we should look at IVF more simply. J Bras Reprod Assist. 2016;20:1-2. PMID: 27203297 DOI: 10.5935/1518-0557.20160001

Devroey $P$, Boostanfar R, Koper NP, Mannaerts BM, Ijzerman-Boon PC, Fauser BC; ENGAGE Investigators. A double-blind, non-inferiority RCT comparing corifollitropin alfa and recombinant FSH during the first seven days of ovarian stimulation using a $\mathrm{GnRH}$ antagonist protocol. Hum Reprod. 2009;24:3063-72. PMID: 19684043 PMCID: PMC2777786 DOI: 10.1093/humrep/dep291

Griesinger G, Boostanfar R, Gordon K, Gates D, McCrary Sisk C, Stegmann BJ. Corifollitropin alfa versus recombinant follicle-stimulating hormone: an individual patient data meta-analysis. Reprod Biomed Online. 2016;33:56-60. PMID: 27178762 DOI: 10.1016/j.rbmo.2016.04.005
Kolibianakis EM, Albano C, Kahn J, Camus M, Tournaye H, Van Steirteghem AC, Devroey P. Exposure to high levels of luteinizing hormone and estradiol in the early follicular phase of gonadotropin-releasing hormone antagonist cycles is associated with a reduced chance of pregnancy. Fertil Steril. 2003;79:873-80. PMID: 12749423 DOI: http://dx.doi.org/10.1016/S0015-0282(02)04920-8

Kolibianakis EM, Venetis CA, Bosdou JK, Zepiridis L, Chatzimeletiou K, Makedos A, Masouridou S, Triantafillidis S, Mitsoli A, Tarlatzis BC. Corifollitropin alfa compared with follitropin beta in poor responders undergoing ICSI: a randomized controlled trial. Hum Reprod. 2015;30:43240. PMID: 25492411 DOI: 10.1093/humrep/deu301

Mahmoud Youssef MA, van Wely M, Aboulfoutouh I, ElKhyat W, van der Veen $\mathrm{F}, \mathrm{Al}$-Inany $\mathrm{H}$. Is there a place for corifollitropin alfa in IVF/ICSI cycles? A systematic review and meta-analysis. Fertil Steril. 2012;97:876-85. PMID: 22277766 DOI: 10.1016/j.fertnstert.2012.01.092

Mardešič T, Mannaerts B, Abuzeid M, Levy $M$, Witjes $H$, Fauser BC; Engage investigators. Short follicular phase of stimulation following corifollitropin alfa or daily recombinant FSH treatment does not compromise clinical outcome: a retrospective analysis of the Engage trial. Reprod Biomed Online. 2014;28:462-8. PMID: 24581989 DOI: $10.1016 /$ j.rbmo.2013.12.009

Orvieto R, Meltzer S, Rabinson J, Zohav E, Anteby EY, Nahum R. GnRH agonist versus $\mathrm{GnRH}$ antagonist in ovarian stimulation: the role of endometrial receptivity. Fertil Steril. 2008;90:1294-6. PMID: 18178197 DOI: $10.1016 /$ j.fertnstert.2007.10.022

Orvieto R, Patrizio P. GnRH agonist versus $\mathrm{GnRH}$ antagonist in ovarian stimulation: an ongoing debate. Reprod Biomed Online. 2013;26:4-8. PMID: 23186555 DOI: $10.1016 /$ j.rbmo.2012.11.001

Rajkhowa M, McConnell A, Thomas GE. Reasons for discontinuation of IVF treatment: a questionnaire study. Hum Reprod. 2006;21:358-63. PMID: 16269448 DOI: $10.1093 /$ humrep/dei355

Requena A, Cruz M, Collado D, Izquierdo A, Ballesteros A, Muñoz M, García-Velasco JA. Evaluation of the degree of satisfaction in oocyte donors using sustained-release FSH corifollitropin a. Reprod Biomed Online. 2013;26:253-9. PMID: 23352098 DOI: 10.1016/j.rbmo.2012.11.015

Westergaard LW, Bossuyt PM, Van der Veen F, van Wely M. WITHDRAWN: Human menopausal gonadotropin versus recombinant follicle stimulation hormone for ovarian stimulation in assisted reproductive cycles. Cochrane Database Syst Rev. 2011;CD003973. PMID: 21328264 DOI: $10.1002 / 14651858 . C D 003973 . p u b 2$ 